DOI:

10.1038/nrc2133

\section{URLs}

EGFR

http://www.ncbi.nlm.nih.gov/

entrez/query.fcgi?db=gene\&c

$\mathrm{md}=$ Retrieve\&dopt=full_

report\&list_uids=1956

NSCLC

http://www.cancer.gov/

cancertopics/types/lung

\section{BCR}

http://www.ncbi.nlm.nih.gov/ entrez/query.fcgi?db=gene\&c $\mathrm{md}=$ Retrievegdopt=full report\&list_uids $=613$

$\mathrm{ABL}$

http://www.ncbi.nlm.nih.gov/ entrez/query.fcgi?db=gene\&c $\mathrm{md}=$ Retrieve\&dopt=full reportElist_uids $=25$

THERAPY

\title{
Crystal-clear targets?
}

The identification of mutations in the epidermal growth factor receptor (EGFR) that account for a subset of patients with non-small-cell lung cancer (NSCLC) prompted the development of several EGFR-targeted tyrosine kinase inhibitors (TKIs). Despite their initial success, patients with NSCLC often develop resistance to EGFR-TKIs within 6-12 months owing to the acquisition of additional resistance-conferring mutations. With a view to improving EGFR TKIs, Michael Eck and colleagues have now characterized the structure of the complexes between a selection of TKIsensitivity-conferring EGFR mutants and TKIs.

The authors generated 12 different crystal structures of mutant EGFR proteins (EGFR-L858R and EGFR-G719S) and wild-type EGFR (EGFR-WT), in complex with the TKIs gefitinib, AEE788 or a staurosporine derivative, AFN941; or in complex with non-hydrolysable ATP. The mutant EGFR proteins in complex with the ATP derivative showed close structural similarity to the activated conformation of EGFR-WT, in agreement with the hypothesis that the substituted amino acids in the mutant proteins destabilize the inactive conformation. Next, the authors assessed the mutant EGFR protein activities in vitro and showed that EGFR-L858R and EGFR-G719S were 50 -fold and 10 -fold more active than EGFR-WT, respectively, further emphasizing the tumorigenic role of these EGFR mutations.

So, can different EGFR mutations confer differences in inhibitor binding? They showed that EGFR-WT,
EGFR-L858R and EGFR-G719S formed structurally similar complexes with gefitinib or AEE788. However, biochemical analyses determined that both gefitinib and AEE788 are significantly more potent against EGFR-L858R and EGFRG719S compared with EGFR-WT, indicating that the EGFR mutations determine the inhibitor potency. Indeed, gefitinib preferentially bound the EGFR-L858R mutant owing to a unique inhibitor-binding mode determined by the mutation. Furthermore, AFN941 showed a unique binding mode to the EGFRG719S mutant.

In contrast to imatinib, the first molecularly targeted therapy to be approved for cancer patients, which selectively targets the kinase domain of BCR-ABL, EGFR TKIs must inhibit many EGFR mutants that show structural variation of the kinase domain and therefore determine the efficacy of inhibitor binding. This paper by Eck and colleagues, published in Cancer Cell, provides a platform from which mutation-specific EGFR-TKIs could be developed that confer improved therapeutic efficacy and reduced patient toxicity owing to the preferential binding to mutant EGFRs. In addition, it will be intriguing to characterize the structural differences of resistance-associated EGFR mutants, such as EGFR-T790M, which have a dominant effect over TKI sensitivity-conferring mutations and have thwarted the clinical use of EGFR TKIs, such as gefitinib, in the US and Europe.

Gemma Alderton
ORIGINAL RESEARCH PAPER Yun, C.-H. et al. Structures of lung cancer-derived EGFR mutants and inhibitor complexes: mechanism of activation and insights into differential inhibitor sensitivity. Cancer Cell 11, 217-227 (2007) FURTHER READING Sharma, S. V. et al. Epidermal growth factor receptor mutations in lung cancer. Nature Rev. Cancer 7, 169-181 (2007)

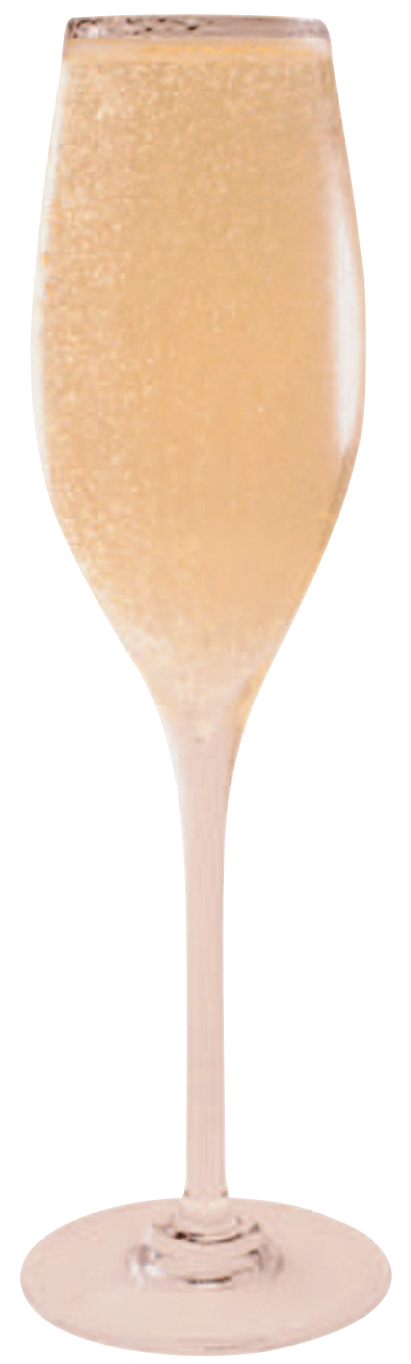

